\title{
WHEN CAN THE MINIMUM VOLUME ENCLOSING SIMPLEX IDENTIFY THE ENDMEMBERS CORRECTLY WHEN THERE IS NO PURE PIXEL?
}

\author{
Wing-Kin Ma ${ }^{\dagger}$, Chia-Hsiang Lin*, Wei-Chiang Li*, and Chong-Yung Chi* \\ $\dagger$ Department of Electronic Engineering, The Chinese University of Hong Kong, Hong Kong \\ * Institute of Communications Engineering, National Tsing Hua University, Hsinchu, Taiwan \\ E-mail: wkma@ieee.org; \{chiahsiang.steven.lin, weichiangli\}@gmail.com; cychi@ee.nthu.edu.tw
}

\begin{abstract}
In blind hyperspectral unmixing, it has been commonly believed that the minimum volume enclosing simplex (MVES) criterion is robust against lack of pure pixels. Specifically, such a belief has been based on empirical experience, where extensive numerical results showed that MVES-based algorithms may identify the underlying endmembers quite accurately under high signal-to-noise ratios and without pure pixels. In this paper, we report some theoretical results on the endmember identifiability of the MVES criterion in the noiseless case. We employ an assumption that is a two-mixture generalization of the pure-pixel assumption; particularly, we require a set of pixels, each being constituted by only two endmembers (rather than one as in the pure-pixel assumption), to exist in the data set. Under this assumption and some rather mild condition, we show that the MVES solution perfectly identifies the true endmembers. Numerical simulation results are provided to verify our theoretical results.
\end{abstract}

Index Terms - Hyperspectral unmixing, minimum volume enclosing simplex, identifiability analysis, convex geometry

\section{INTRODUCTION}

Hyperspectral unmixing (HU) is a crucial technology for extracting the underlying materials (or endmembers) and their corresponding abundance maps in a scene of interest from the observed hyperspectral imaging data $[1,2]$. It has numerous applications such as mineral identification, environmental monitoring, to name a few. In this context, the well-known pure-pixel assumption has been very successfully utilized for the development of blind HU algorithms; such pure-pixel-based algorithms are not only easy to implement, their performance is also promising when the pure-pixel assumption holds. However, the pure-pixel assumption may be too restrictive (at least in an exact sense) when dealing with heavily mixed scenarios. In the no pure-pixel case, another approach, called minimum volume enclosing simplex (MVES), and also known as minimum volume simplex analysis (MVSA) or simplex volume minimization, has been found to exhibit robustness against lack of pure pixels [3-5]. Advocated by Craig in the early 1990's in the context of remote sensing [6], the idea is to find a set of vertices whose simplex encloses the data cloud and whose simplex volume is the smallest.

To give some insight, in Fig. 1 we provide demonstrations of the MVES for the case of three endmembers and noiseless data. Fig. 1(a) illustrates an instance where pure pixels are missing, and

This work was supported partly by a General Research Fund of Hong Kong Research Grant Council (Project No. CUHK4441269), and partly by National Science Council (R.O.C.) under Grant NSC 102-2221-E-007-035MY2.

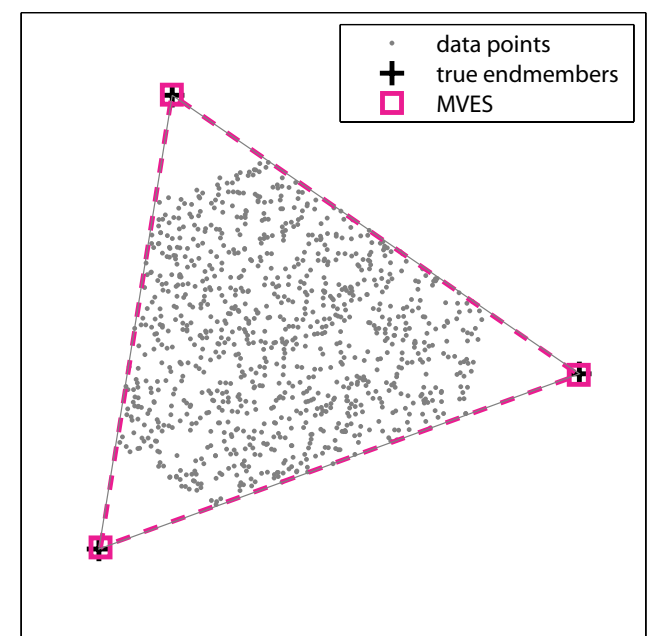

(a)

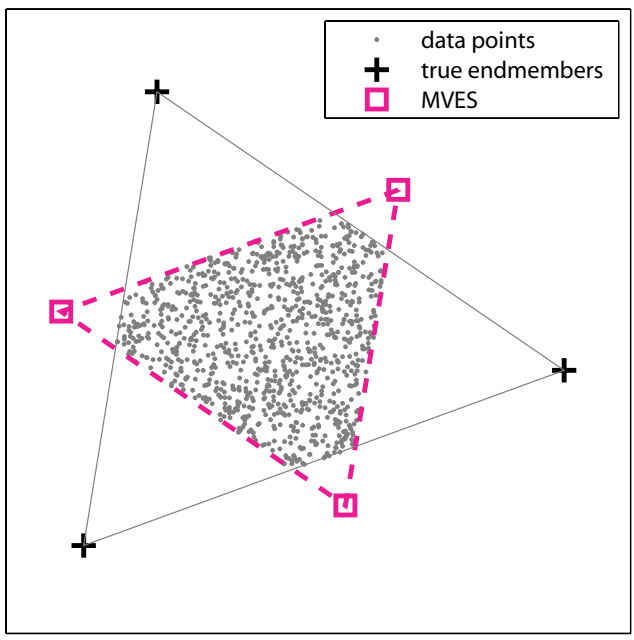

(b)

Fig. 1. Demonstrations of the MVES for the case of three endmembers and noiseless data, where MVES works well in the scenario (a) and fails in the scenario (b).

we can see that the MVES estimates the true endmembers very accurately. Fig. 1(b) illustrates another instance where the data points become very heavily mixed and there is no pure pixel, and this time 
the MVES performs poorly. Hence, at least from the above demonstrations, it seems that the MVES can be robust against lack of pure pixels if the data points are not too heavily mixed.

We should note that from a viewpoint of computational complexity, the MVES criterion amounts to an optimization problem that is known to be NP-hard in general [7]. Despite such a limitation, several practical algorithms for handling the MVES problem, e.g., [3-5], have been developed, and numerical results therein have demonstrated that the MVES-based algorithms may give reasonably accurate endmember identification performance under high signal-to-noise ratios and without pure pixels. Such a finding was encouraging, and has suggested an opportunity for robust blind $\mathrm{HU}$ solutions in the no pure-pixel regime. From a theoretical viewpoint, it also leads to the following question:

Assume the noiseless case. Is it possible to prove when the MVES solution identifies the true endmembers perfectly? If such results can be proven, what are the conditions that the data should satisfy to attain perfect identifiability?

An answer to the above question is not only meaningful in a theoretical sense, but the subsequent results would also benefit the advances of blind HU techniques in remote sensing.

Very recently, we endeavor to address the above question by proving several MVES endmember identifiability results in the noiseless case [8]. This conference paper serves as an abridged version of [8]. We will focus on reporting one key result in [8], where we consider an assumption that may roughly be regarded as an "order-two" generalization of the pure-pixel assumption. From this assumption, we will show when the MVES solution identifies the true endmembers exactly and uniquely. The practical implications of the identifiability result will be discussed, and numerical results for verifying the result will be provided.

Some basic notations are as follows. The set of all real-valued $n$-dimensional vectors and $m$-by- $n$ matrices are denoted by $\mathbb{R}^{n}$ and $\mathbb{R}^{m \times n}$, respectively (resp.); $\|\cdot\|$ denotes the Euclidean norm of a vector; $\boldsymbol{x}^{T}$ denotes the transpose of the vector $\boldsymbol{x} ; \boldsymbol{x} \geq \boldsymbol{0}$ means that $\boldsymbol{x}$ is elementwise non-negative; $\mathbf{1}$ denotes an all-one vector of appropriate dimension.

\section{PROBLEM STATEMENT AND BACKGROUND}

The MVES identifiability problem considered in this paper is based on a conventional blind $\mathrm{HU}$ problem setup; see the literature, e.g., $[1,2]$, for full coverage. The signal model is briefly described as follows. Let $\boldsymbol{x}_{n} \in \mathbb{R}^{M}$ denote the $n$th pixel of a hyperspectral image captured at a scene of interest, where the elements of $\boldsymbol{x}_{n}$ are spectral measurements at different bands, and $M$ is the number of spectral bands. The pixels are modeled as linear mixtures of the spectral signatures of the underlying endmembers in the scene, and, in the noiseless case, the corresponding mixing model is written as

$$
\boldsymbol{x}_{n}=\boldsymbol{A} \boldsymbol{s}_{n}, \quad n=1, \ldots, L,
$$

where $\boldsymbol{A}=\left[\boldsymbol{a}_{1}, \ldots, \boldsymbol{a}_{N}\right] \in \mathbb{R}^{M \times N}$ is the endmember signature matrix, with each column $\boldsymbol{a}_{i}$ being the spectral signature vector of a distinct endmember; $N$ is the number of endmembers; $\boldsymbol{s}_{n} \in \mathbb{R}^{N}$ is the abundance vector at the $n$th pixel; $L$ is the number of pixels. As standard blind $\mathrm{HU}$ assumptions, we have the following assumptions:

i) every abundance vector satisfies $\boldsymbol{s}_{n} \geq 0, \mathbf{1}^{T} \boldsymbol{s}_{n}=1$ (i.e., the abundance non-negativity and sum-to-one constraints),

ii) $\boldsymbol{A}$ has full column rank, and iii) $\left[s_{1}, \ldots, s_{L}\right]$ has full row rank

The MVES is a blind HU approach that attempts to recover the unknown endmember spectral signatures $\boldsymbol{a}_{1}, \ldots, \boldsymbol{a}_{N}$ from the data $\boldsymbol{x}_{1}, \ldots, \boldsymbol{x}_{L}$ by finding an $(N-1)$-dimensional simplex that circumscribes all the data points $\boldsymbol{x}_{n}$ 's and yields the smallest volume. To put this into context, let

$$
\operatorname{conv}\left\{\boldsymbol{b}_{1}, \ldots, \boldsymbol{b}_{N}\right\}=\left\{\boldsymbol{x}=\sum_{i=1}^{N} \theta_{i} \boldsymbol{b}_{i} \mid \boldsymbol{\theta} \geq \mathbf{0}, \mathbf{1}^{T} \boldsymbol{\theta}=1\right\},
$$

denote the convex hull of $\left\{\boldsymbol{b}_{1}, \ldots, \boldsymbol{b}_{N}\right\} \subseteq \mathbb{R}^{M}$, and vol $\mathcal{A}$ denote the volume of the given set $\mathcal{A}$. The MVES criterion can be formulated as an optimization problem

$$
\begin{aligned}
\underset{\boldsymbol{b}_{1}, \ldots, \boldsymbol{b}_{N} \in \mathbb{R}^{M}}{\min } & \operatorname{vol}\left(\operatorname{conv}\left\{\boldsymbol{b}_{1}, \ldots, \boldsymbol{b}_{N}\right\}\right) \\
\text { s.t. } & \boldsymbol{x}_{n} \in \operatorname{conv}\left\{\boldsymbol{b}_{1}, \ldots, \boldsymbol{b}_{N}\right\}, n=1, \ldots, L,
\end{aligned}
$$

where the solution to problem (2) serves as an estimate of the endmember spectral signatures. Note that in problem (2), the number of endmembers $N$ is assumed to be known. As discussed in the Introduction, problem (2) is NP-hard in general [7]. This means that the existing MVES-based algorithms [3-5], which were developed from an application-driven viewpoint, may not always obtain the solution to problem (2) in a globally optimal sense. Despite that, practical experience has suggested that the MVES-based algorithms may give reasonable endmember identification performance-including instances where the pure-pixel assumption is violated.

Motivated by the good empirical results with the MVES-based algorithms, in this paper we consider the theoretical perfect identifiability of the MVES criterion in problem (2). Specifically, we define perfect identifiability as conditions under which the solution to problem (2) is uniquely and exactly given by the true endmember spectral signatures $\boldsymbol{a}_{1}, \ldots, \boldsymbol{a}_{N}$, subject to permutations of the ordering of $\boldsymbol{a}_{1}, \ldots, \boldsymbol{a}_{N}$, which are minor effects. Before proceeding to the main result in the next section, we should mention an existing MVES identifiability result. To describe it, let us recall the definition of the pure-pixel assumption.

Assumption 1 (pure-pixel assumption) For every $i \in\{1, \ldots$, $N\}$, there exists a pixel, whose index is denoted by $n(i)$, such that its abundance vector takes the form

$$
\boldsymbol{s}_{n(i)}=\boldsymbol{e}_{i}
$$

where $\boldsymbol{e}_{i}$ denotes a unit vector, with $\left[\boldsymbol{e}_{i}\right]_{j}=1$ for $j=i$ and $\left[\boldsymbol{e}_{i}\right]_{j}=$ 0 for $j \neq i$.

The corresponding MVES identifiability result is as follows.

Fact 1 ( [4], Theorem 1) Assume the noiseless case, and suppose that Assumption 1 holds. Then, the solution to problem (2) is uniquely and exactly given by $\boldsymbol{a}_{1}, \ldots, \boldsymbol{a}_{N}$ subject to ordering permutations.

\section{THE MAIN RESULT}

In this section, we report an MVES perfect identifiability result in the no pure-pixel case. We consider an assumption that is more general than the pure-pixel assumption, given as follows. 
Assumption 2 For every $i, j \in\{1, \ldots, N\}, i \neq j$, there exists a pixel, whose index is denoted by $n(i, j)$, such that its abundance vector takes the form

$$
\boldsymbol{s}_{n(i, j)}=\alpha_{i j} \boldsymbol{e}_{i}+\left(1-\alpha_{i j}\right) \boldsymbol{e}_{j},
$$

for some coefficient $\alpha_{i j}$ that satisfies $\frac{1}{2}<\alpha_{i j} \leq 1$.

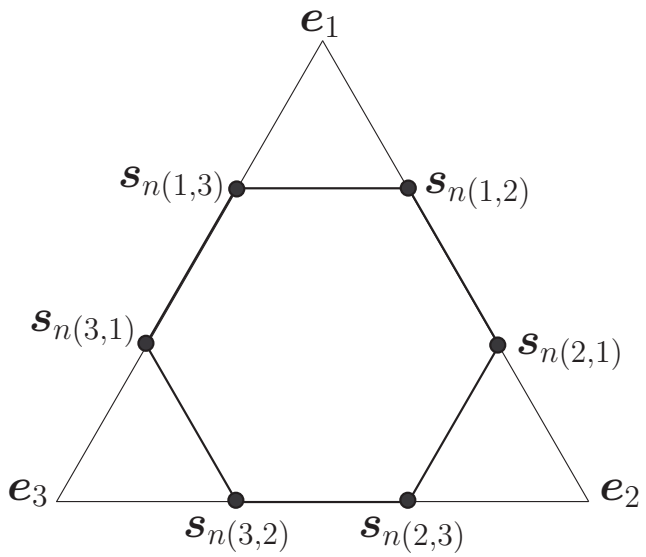

Fig. 2. Illustration of Assumption 2 for the case of $N=3$.

Fig. 2 gives an illustration of Assumption 2 for the case of $N=3$. Assumption 2 means that one can find pixels that are constituted by two endmembers (for all pairs of endmembers), with one dominating another as indicated by the coefficient $\alpha_{i j}>\frac{1}{2}$. Also, the pixels in (3) lie on the edges of the unit simplex. In addition, Assumption 2 is seen to reduce to Assumption 1 when $\alpha_{i j}=1$ for all $i, j$. Hence, Assumption 2 subsumes the pure-pixel assumption. From Assumption 2, we have the following main result.

Claim 1 Assume the noiseless case, and suppose that Assumption 2 holds. Then, the solution to problem (2) is uniquely and exactly given by $\boldsymbol{a}_{1}, \ldots, \boldsymbol{a}_{N}$ subject to ordering permutations if

1. $N=3$ and $\alpha_{i j}>\frac{2}{3}$ for all $i, j$, or if

2. $N \geq 4$.

Claim 1 is a consequence of two theorems in [8]. To get some feeling with Claim 1, take the demonstrations back in Fig. 1 as an example. The two instances in Fig. 1 basically satisfy Assumption 2 The instance in Fig. 1(a) has $\alpha_{i j}>\frac{2}{3}$, while that in Fig. 1(b) has $\alpha_{i j}<\frac{2}{3}$. The identifiability results observed in these two instances have a good match with that in condition 1 of Claim 1.

Let us discuss the implications arising from Claim 1. First, Claim 1 suggests that if the pixels are not too heavily mixed so that there exist "good" pixels that are at appropriate positions on the edge of the unit simplex, it is possible for the MVES to attain perfect identifiability. Second, we observe that the perfect identifiability result for $N \geq 4$ has no restriction on the mixing coefficients $\alpha_{i j}$ 's (as long as they satisfy $\frac{1}{2}<\alpha_{i j} \leq 1$ ), and is more relaxed than that for $N=3$. This seems to suggest that the MVES criterion may provide stronger identifiability as the problem dimension $N$ increases. However, we should also note that there is no free lunch. To satisfy Assumption 2 with general $\alpha_{i j}$ 's, we would need at least $N(N-1)$ pixels in (3). Subsequently, the number of pixels $L$ should be no less than $N(N-1)$, which implies that more pixels would be required as the problem dimension increases.

\section{SKETCH OF THE PROOF OF THE MAIN RESULT}

In this section we give a sketch of the proof of Claim 1. Readers can find the full details in [8]. For convenience, let

$$
\mathcal{X}_{L}=\left\{\boldsymbol{x}_{1}, \ldots, \boldsymbol{x}_{L}\right\}, \quad \mathcal{S}_{L}=\left\{\boldsymbol{s}_{1}, \ldots, \boldsymbol{s}_{L}\right\},
$$

denote the sets of all the observed pixels and the corresponding abundance vectors, resp., and

$$
\begin{aligned}
& \mathcal{T}_{e}=\operatorname{conv}\left\{\boldsymbol{e}_{1}, \ldots, \boldsymbol{e}_{N}\right\} \subseteq \mathbb{R}^{N}, \\
& \mathcal{T}_{a}=\operatorname{conv}\left\{\boldsymbol{a}_{1}, \ldots, \boldsymbol{a}_{N}\right\} \subseteq \mathbb{R}^{M},
\end{aligned}
$$

be the $(N-1)$-dimensional unit simplex and the true endmembers' simplex, resp. Consider the following definition.

Definition 1 (minimum volume enclosing simplex) Given an $m$ dimensional ${ }^{1}$ set $\mathcal{U} \subseteq \mathbb{R}^{n}$, MVES $(\mathcal{U})$ denotes the set that collects all $m$-dimensional minimum volume simplices that enclose $\mathcal{U}$ and lie in the affine hull of $\mathcal{U}$.

From the above definition, the perfect MVES identifiability problem can be stated as the problem of finding conditions under which

$$
\operatorname{MVES}\left(\mathcal{X}_{L}\right)=\left\{\mathcal{T}_{a}\right\}
$$

As our first step, consider the following result.

Proposition $1 \operatorname{MVES}\left(\mathcal{X}_{L}\right)=\left\{\mathcal{T}_{a}\right\}$ if and only if $\operatorname{MVES}\left(\mathcal{S}_{L}\right)=$ $\left\{\mathcal{T}_{e}\right\}$.

The proof of Proposition 1, as well as those of the theorems to be presented below, can be found in [8], and here we omit the details. By Proposition 1, the perfect MVES identifiability problem reduces to that of finding conditions under which

$$
\operatorname{MVES}\left(\mathcal{S}_{L}\right)=\left\{\mathcal{T}_{e}\right\}
$$

which is invariant with $\boldsymbol{a}_{1}, \ldots, \boldsymbol{a}_{N}$.

Our second step aims at finding a relationship between the condition $\operatorname{MVES}\left(\mathcal{S}_{L}\right)=\left\{\mathcal{T}_{e}\right\}$ and the abundance pixel purities. To this end, we introduce the following measure

$$
\gamma=\sup \left\{r \leq 1 \mid \mathcal{R}(r) \subseteq \operatorname{conv}\left\{\boldsymbol{s}_{1}, \ldots, \boldsymbol{s}_{L}\right\}\right\},
$$

where

$$
\mathcal{R}(r)=\left\{\boldsymbol{s} \in \operatorname{conv}\left\{\boldsymbol{e}_{1}, \ldots, \boldsymbol{e}_{N}\right\} \mid\|\boldsymbol{s}\| \leq r\right\} .
$$

We call (4) the uniform pixel purity level. Owing to space limitation, we shall not describe the physical meanings of (4) in detail. Interested readers are referred to [8], wherein we give geometric interpretations of (4). In simple terms, (4) quantifies a pixel purity level that applies to all the endmembers. In particular, it can be shown that $\frac{1}{\sqrt{N}} \leq \gamma \leq 1$, where the most heavily mixed instance $\boldsymbol{s}_{1}=\cdots=\boldsymbol{s}_{L}=\frac{1}{N} \mathbf{1}$ has $\gamma=\frac{1}{\sqrt{N}}$, and the pure-pixel instances (i.e., instances that satisfy Assumption 1) have $\gamma=1$. A key result arising from the uniform pixel purity level is as follows.

Theorem 1 Assume $N \geq 3$. If the uniform pixel purity level satisfies

$$
\gamma>\frac{1}{\sqrt{N-1}}
$$

then $\operatorname{MVES}\left(\mathcal{S}_{L}\right)=\left\{\mathcal{T}_{e}\right\}$.

\footnotetext{
${ }^{1}$ Here, the dimension of a set $\mathcal{A} \subseteq \mathbb{R}^{n}$ is defined as the affine dimension of the affine hull of $\mathcal{A}$.
} 
The last step lies in linking the conditions in Theorem 1 and Assumption 2.

Theorem 2 Under Assumption 2 and for $N \geq 2$, the uniform pixel purity level satisfies

$$
\gamma \geq \sqrt{\frac{1}{N}\left[\frac{(N \alpha-1)^{2}}{N-1}+1\right]},
$$

where $\alpha=\min _{i, j \in\{1, \ldots, N\}, i \neq j} \alpha_{i j}$.

If we compare Theorems 1 and 2, we see that the condition

$$
\sqrt{\frac{1}{N}\left[\frac{(N \alpha-1)^{2}}{N-1}+1\right]}>\frac{1}{\sqrt{N-1}},
$$

implies perfect identifiability of MVES. It is shown that the above inequality is equivalent to

$$
\alpha>\frac{2}{N},
$$

for $N \geq 3$. By also noting $\frac{1}{2}<\alpha \leq 1$ in Assumption 2, and the fact that $\frac{1}{2} \geq \frac{2}{N}$ for $N \geq 4$, we obtain Claim 1 .

Before we finish, we should give a remark. From the proof above, we see that Claim 1 is a special case of Theorem 1. As a future direction, it would be interesting to study whether there are other conditions that also satisfy Theorem 1; by doing so we may pin down more MVES perfect identifiability results.

\section{NUMERICAL SIMULATIONS}

In this section, we verify Claim 1 through numerical simulations. We generate the data points $\boldsymbol{x}_{n}$ 's by the noiseless linear mixing model in (1). Since perfect MVES identifiability does not depend on the endmember signatures in the noiseless case (cf. Proposition 1), we simply choose $\left\{\boldsymbol{a}_{1}, \ldots, \boldsymbol{a}_{N}\right\}=\left\{\boldsymbol{e}_{1}, \ldots, \boldsymbol{e}_{N}\right\}$ and $M=N$. The abundance pixels $\boldsymbol{s}_{n}$ 's are generated according to Assumption 2. Specifically, we set $\alpha_{i j}=\alpha$ for all $i, j$, generate all the combinations of (3), and use them as the abundance pixel set.

We implement the MVES by using the alternating linear programming (LP) algorithm in [4]. This algorithm does not guarantee convergence to the MVES solution in a globally optimal sense (note that the same applies to the other computationally efficient MVES-based algorithms, as the MVES problem is generally NPhard). We improve the chance of hitting the MVES solution by running the alternating LP algorithm 20 times, each with a different initial point, and then choosing the result that gives the smallest simplex volume. The endmember identification performance of the resulting endmember estimate is evaluated by using the root-meansquare (RMS) angle error

$$
\phi=\min _{\boldsymbol{\pi} \in \Pi_{N}} \sqrt{\frac{1}{N} \sum_{i=1}^{N}\left[\arccos \left(\frac{\boldsymbol{a}_{i}^{T} \hat{\boldsymbol{a}}_{\pi_{i}}}{\left\|\boldsymbol{a}_{i}\right\| \cdot\left\|\hat{\boldsymbol{a}}_{\pi_{i}}\right\|}\right)\right]^{2}},
$$

where $\Pi_{N}$ denotes the set of all permutations of $\{1, \ldots, N\}$, and $\left\{\hat{\boldsymbol{a}}_{1}, \ldots, \hat{\boldsymbol{a}}_{N}\right\}$ denotes the endmember estimate.

Fig. 3 shows the RMS angle errors with respect to $\alpha$. Note that a smaller $\alpha$ means more heavily mixed pixels, and vice versa. We can see that for $N \geq 4$, zero RMS angle error, or equivalently, perfect MVES identifiability, is attained for any $\frac{1}{2}<\alpha \leq 1$. Also, for $N=3$, zero RMS angle error is attained for $\frac{2}{3}<\alpha \leq 1$. These observations are in good agreement with Claim 1.

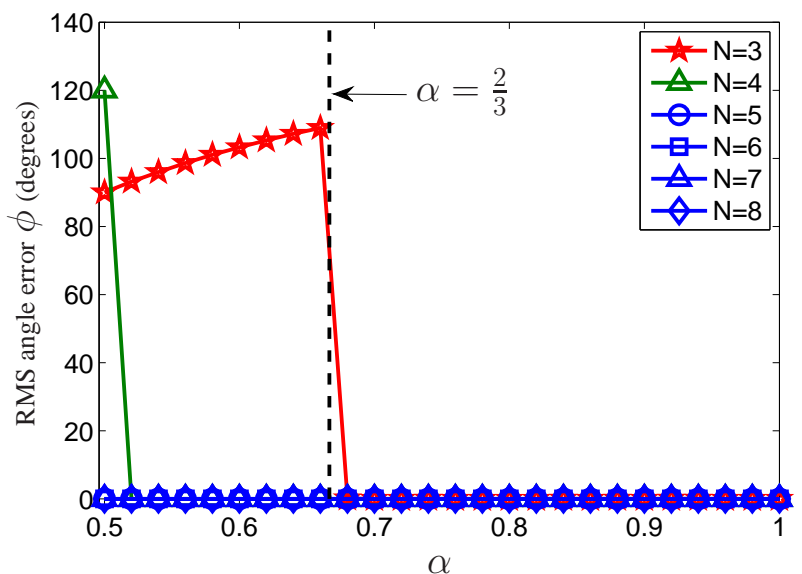

Fig. 3. A numerical result on the MVES identification performance.

\section{CONCLUSIONS AND DISCUSSIONS}

In this paper, we have presented a sufficient condition for the MVES to attain perfect endmember identifiability in the noiseless case; cf. Claim 1. The condition considers a two-mixture generalization of the well-known pure-pixel assumption, and suggests that the MVES is robust against lack of pure pixels to a certain level. As future work, it would be interesting to study how the analysis leading to the above condition can be extended to provide further MVES identifiability results; e.g., those in the noisy case.

\section{REFERENCES}

[1] J. Bioucas-Dias, A. Plaza, N. Dobigeon, M. Parente, Q. Du, P. Gader, and J. Chanussot, "Hyperspectral unmixing overview: Geometrical, statistical, and sparse regression-based approaches," IEEE J. Sel. Topics Appl. Earth Observ., vol. 5, no. 2, pp. 354-379, 2012.

[2] W.-K. Ma, J. M. Bioucas-Dias, T.-H. Chan, N. Gillis, P. Gader, A. J. Plaza, A. Ambikapathi, and C.-Y. Chi, "A signal processing perspective on hyperspectral unmixing," IEEE Signal Process. Mag., vol. 31, no. 1, pp. 67-81, 2014.

[3] J. Li and J. Bioucas-Dias, "Minimum volume simplex analysis: A fast algorithm to unmix hyperspectral data," in Proc. IEEE IGARSS, Aug. 2008

[4] T.-H. Chan, C.-Y. Chi, Y.-M. Huang, and W.-K. Ma, "A convex analysis based minimum-volume enclosing simplex algorithm for hyperspectral unmixing," IEEE Trans. Signal Process., vol. 57, no. 11, pp. 4418-4432, 2009.

[5] J. Bioucas-Dias, "A variable splitting augmented Lagrangian approach to linear spectral unmixing," in Proc. IEEE WHISPERS, Aug. 2009.

[6] M. D. Craig, "Minimum-volume transforms for remotely sensed data," IEEE Trans. Geosci. Remote Sens., vol. 32, no. 3, pp. 542-552, May 1994.

[7] A. Packer, "NP-hardness of largest contained and smallest containing simplices for V- and H-polytopes," Discrete and Computational Geometry, vol. 28, no. 3, pp. 349-377, 2002.

[8] C.-H. Lin, W.-K. Ma, W.-C. Li, C.-Y. Chi, and A. Ambikapathi, "Identifiability of the simplex volume minimization criterion for blind hyperspectral unmixing: The no pure-pixel case," to appear in IEEE Trans. Geosci. Remote Sens., 2015, available on http://arxiv.org/abs/1406.5273. 